\title{
Clinical Trials of Immunomodulation in Ischemic Stroke
}

\author{
Roland Veltkamp ${ }^{1} \cdot$ Dipender Gill $^{1}$
}

Published online: 13 July 2016

(C) The American Society for Experimental NeuroTherapeutics, Inc. 2016

\begin{abstract}
Inflammatory mechanisms are currently considered as a prime target for stroke therapy. There is evidence from animal studies that immune signals and mediators can have both detrimental and beneficial effects in particular stages of the disease process. Moreover, several of these mechanisms are turned on with sufficient delay after ischemia onset to make them amenable to therapeutic intervention. Several clinical proof-of concept trials have investigated the efficacy of different immunomodulatory approaches in patients with stroke. Trials targeting the innate immune system have focused on reduction of microglial activation, inhibition of neutrophil migration, and interleukin-1 receptor blockade, suggesting that interleukin-1 receptor blockade may be a promising strategy. Studies aiming at halting T-cell migration have also been undertaken with controversial findings regarding prevention of infarct growth in neuroimaging studies. Consistently, recent proof-of-concept trials targeting lymphocytes with drugs such as natalizumab and fingolimod have yielded some promising results on clinical endpoints, but confirmation in larger trials is needed. At present, the understanding of the role of immune mechanisms in neurorepair and neurodegeneration is limited. Improving long-term brain function by mitigating prolonged neuroinflammation that was triggered by acute brain injury could be a strategy in addition to neuroprotection.
\end{abstract}

Keywords Immunomodulation · immune system · ischemic stroke $\cdot$ stroke

Roland Veltkamp

r.veltkamp@imperial.ac.uk

1 Department of Stroke Medicine, Imperial College London, London, UK

\section{Introduction}

Ischaemic stroke typically results from thrombotic or thromboembolic blockage of a cerebral artery. Primarily, viability of the ischemic brain depends on the duration and the severity of blood flow reduction because brain energy metabolism is critically dependent on continuous oxygen supply. Rapid restoration of cerebral blood supply by intravenous thrombolysis and, more recently, by mechanical thrombectomy is currently the mainstay of acute stroke therapy $[1,2]$. Beyond failure of aerobic energy metabolism, ischaemia triggers a wide array of secondary molecular, cellular, and systemic processes, many of which substantially increase brain damage in experimental stroke models [3, 4]. Protection of the brain against deleterious processes during or after ischemia has been a main concept in the development of new stroke therapies [5]. So far, however, the results of medical and physical treatments in experimental stroke models have not been translated successfully into the clinical setting $[6,7]$ (Table 1).

Inflammatory mechanisms are currently considered as a prime target for stroke therapy $[3,4]$. The vast number of cellular and molecular processes commonly referred to as inflammatory precludes that inflammation as a whole can be categorized into either good or bad $[3,4,8]$. Nevertheless, there is evidence from animal studies that certain immune signals and mediators have profound detrimental effects, at least in certain stages of the disease process. Moreover, several of these mechanisms are turned on with sufficient delay after ischemia onset to make them amenable to therapeutic intervention. However, there are concerns that immune modulation in patients with stroke may exacerbate poststroke immune depression and result in increased infectious complications [9-12].

Several clinical proof-of concept trials have investigated the efficacy of different immunomodulatory approaches in 


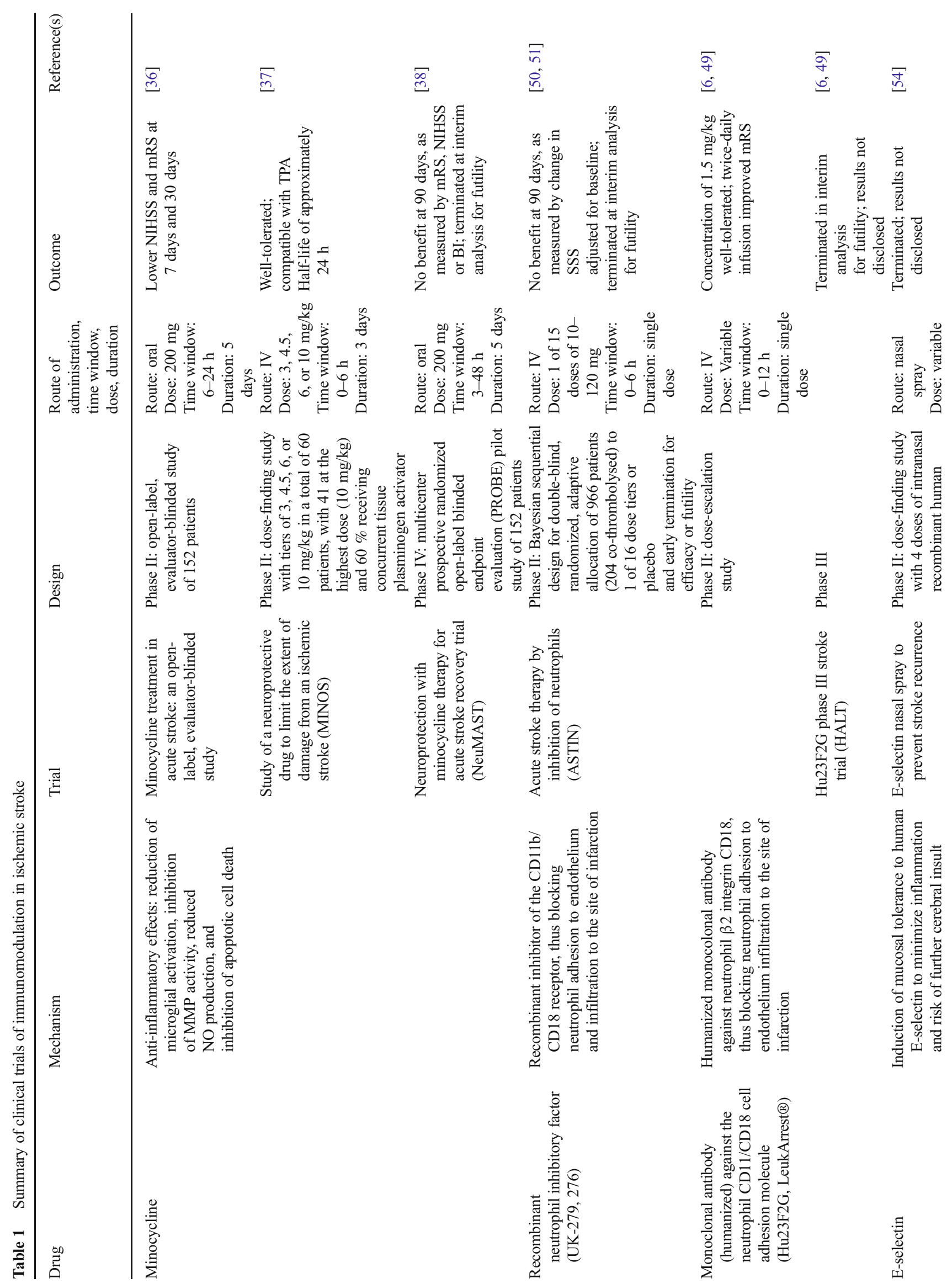




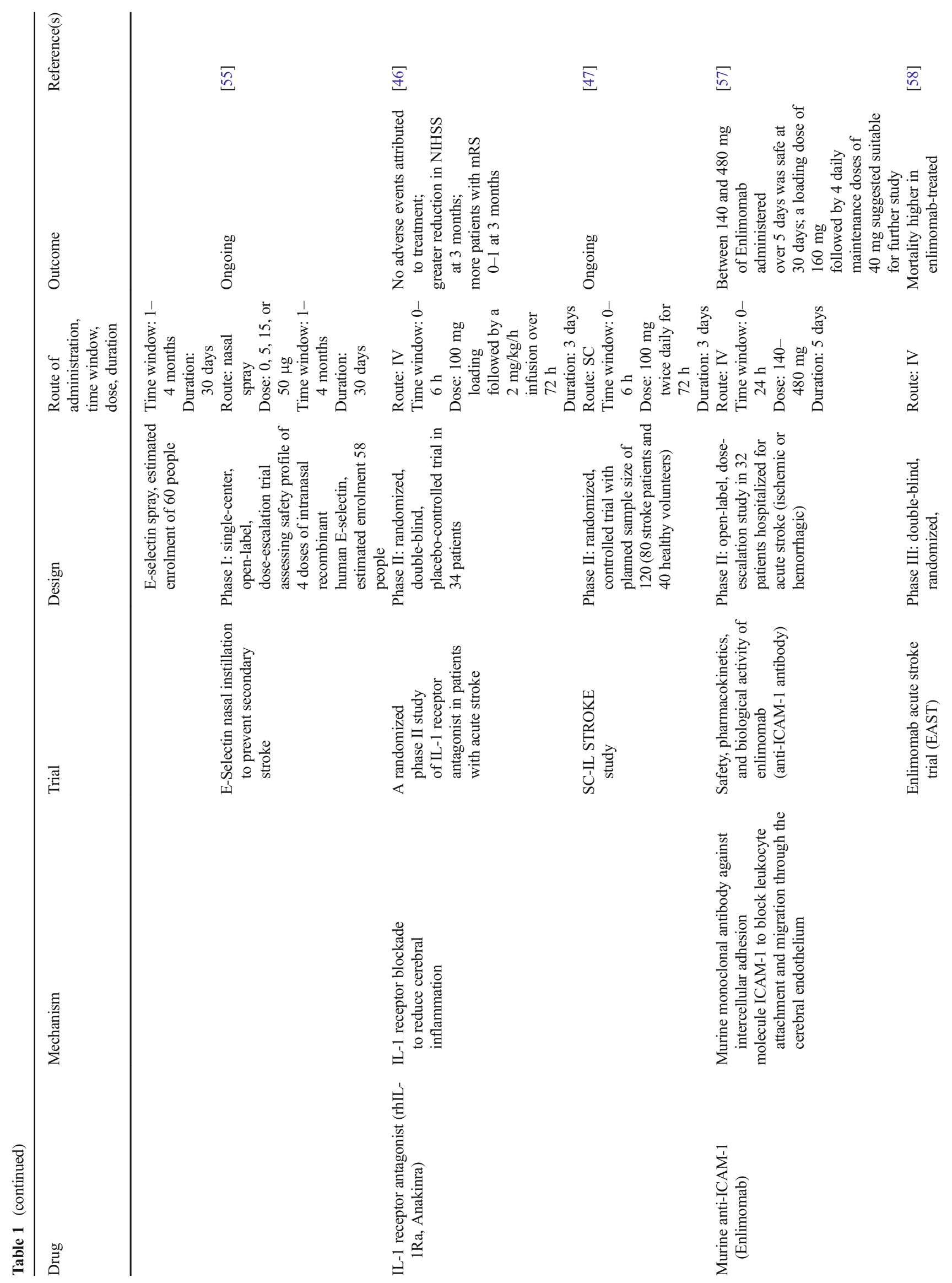




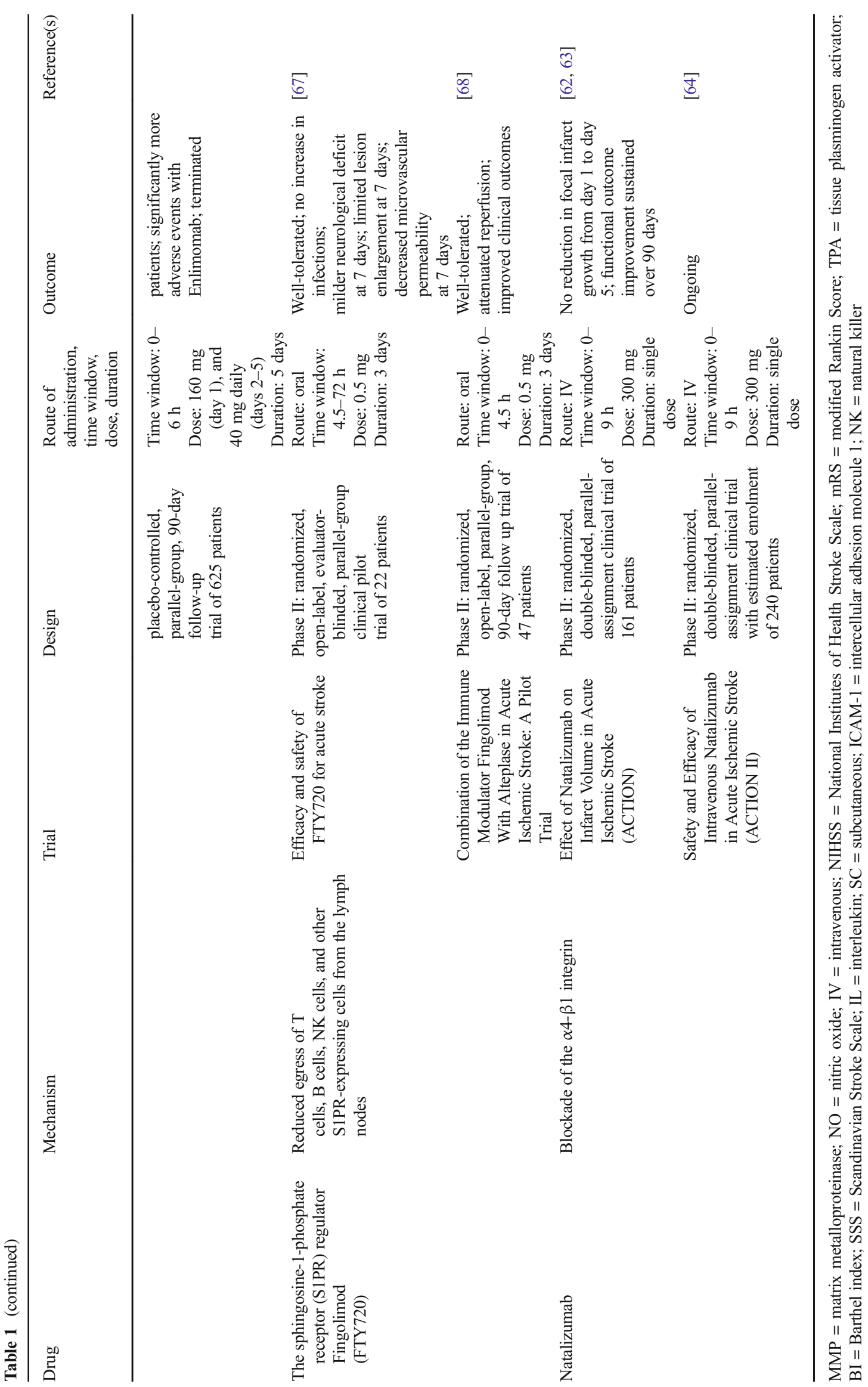


patients with stroke. The present review summarizes the results of previous and ongoing trials targeting different components of the innate and adaptive immune system. Conceptual consequences for future translational research from the findings of these early-phase trials will be drawn.

\section{Immune Mechanisms}

Stroke activates multiple inflammatory cascades in the brain and in the systemic immune system [3, 4, 13]. Upon injury, neurons and other damaged brain cells release a number of molecules that function as danger-associated molecular patterns or alarmins [14]. These mediators bind to pattern recognition receptors on various cells, including microglia and endothelial cells, and lead to their activation [14]. Microglia sense changes in the ischemic brain [15]. They upregulate major histocompatibility complex class II molecules, and express and secrete cytokines, including tumour necrosis factor- $\alpha$ and interleukin (IL)-1 [16]. Activated cerebral microvessels become more permeable to molecules that are normally prevented from crossing the blood-brain barrier [17]. In particular, the immunological blood-brain barrier is substantially altered after ischemia [18]. Together with the secretion of chemokines this promotes the successive entry of systemic leukocytes including neutrophils, macrophages, and lymphocytes $[3,13,19,20]$. Corresponding to upregulation of adhesion molecules on endothelial cells, integrins serve similar functions on activated leukocytes. The sequence of leukocyte recruitment into the brain after experimental stroke has been well characterized [21], whereas the temporal and spatial profile immune cell recruitment after stroke in humans requires better characterization [22].

A substantial role for the adaptive immune response after stroke is increasingly recognized. Transgenic animals deficient in lymphocytes consistently have smaller infarcts in different stroke models [23-26]. Moreover, antibody-mediated depletion of $\mathrm{CD}^{+}, \mathrm{CD} 8^{+}$, and $\gamma \delta \mathrm{T}$ cells reduced infarct volume and improved functional outcome [25, 27-29]. The dynamics of this deleterious role of different proinflammatory $\mathrm{T}$ cells have not been fully elucidated. Interestingly, this effect was evident $24 \mathrm{~h}$ after ischemia onset in some studies, suggesting an antigen-independent effect of T cells. Other studies consistently describe a delayed mechanism of tissue injury. Further evidence points to a role for B cells, though perhaps in a regulatory capacity [30]. Cytokines are key mediators in the inflammatory response to stroke [31]. While recent research provides solid support for a deleterious role for interferon- $\gamma$ in poststroke inflammation [32], evidence for an inciting effect of IL-17 is also mounting, with innate $\gamma \delta$ T cells likely to be a main source [33]. Currently, the target of immunomodulation translated into clinical trials is focused on the early phase of toxic neuroinflammation. However, the neuroinflammatory reaction after acute brain injury continues for months $[4,34,35]$, and the complex effect on repair and degeneration after stroke remains to be unravelled. This further highlights the dualistic nature of the immune response to stroke, acting not only to exacerbate damage with detrimental effects, but also to propagate repair and recovery.

\section{Stroke Trials Addressing Innate Immune Mechanisms}

\section{Microglia as a Target}

Minocycline is a second-generation derivative of tetracycline that has a protective effect in animal models of stroke through a variety of mechanisms, including anti-inflammatory effects, reduction of microglial activation, matrix metalloproteinase reduction, nitric oxide production, and inhibition of apoptotic cell death [36]. An open-label, evaluator-blinded study of 152 patients showed minocycline, when administered orally for 5 days at a dosage of $200 \mathrm{mg}$ within 6 to $24 \mathrm{~h}$ of onset of stroke, to be associated with significantly lower National Institutes of Health Stroke Scale (NIHSS) score and modified Rankin Score (mRS) compared with placebo [36]. This pattern was apparent on day 7 of follow-up, and continued to day 30 [36]. Furthermore, there was no difference in the incidence of observed complications [36]. These findings prompted further study into the safety and dose range of minocycline [37]. In a phase IIb trial, "Minocycline to Improve Neurologic Outcome in Stroke" (MINOS), minocycline was administered intravenously within $6 \mathrm{~h}$ of stroke symptom onset in preset dose tiers of $3,4.5,6$, or $10 \mathrm{mg} / \mathrm{kg}$ daily over $72 \mathrm{~h}$ [37]. A total of 60 patients were recruited, with 41 at the highest dose $(10 \mathrm{mg} / \mathrm{kg})$ and $60 \%$ receiving concurrent thrombolysis with tissue plasminogen activator. Minocycline infusion was well tolerated with only 1 observation of dose limiting toxicity in the $10-\mathrm{mg} / \mathrm{kg}$ regimen. Furthermore, there were no incidences of severe hemorrhage in the thrombolysed patients, confirming compatibility with tissue plasminogen activator [37]. Pharmacokinetic analysis revealed a half-life of approximately $24 \mathrm{~h}$, thus allowing once-daily dosing [37].

These encouraging results prompted a multicentre randomized, double-blind, placebo controlled trial, "Neuroprotection With Minocycline Therapy for Acute Stroke Recovery Trial" (NeuMAST), in which patients with ischemic stroke were randomized to treatment with either oral minocycline or placebo within 3 to $48 \mathrm{~h}$ of symptom onset. The assigned treatment was administered for 5 consecutive days after enrolment, and the primary efficacy endpoint was an mRS of $0-1$ for all randomized patients at 90 days, with secondary endpoints including NIHSS and Barthel Index at 90 days, analysed using ordinal shift analysis. Unfortunately, the study did not show minocycline to have any efficacy in improving long-term 
recovery, and the trial was abandoned in May 2013 after interim analysis suggested futility [38].

\section{Interleukin-1}

IL- 1 is a proinflammatory mediator with 2 main ligands- IL$1 \alpha$ and IL-1 $\beta$ [39] — as well as a third naturally occurring competitive antagonist, IL-1Ra [40]. Stroke causes upregulation of the IL-1 receptor and its ligands in animal models [41], with expression of IL- $1 \alpha$ seen in microglia within $4 \mathrm{~h}$ postreperfusion [42]. Furthermore, exogenous administration of IL-1 $\beta$ exacerbates ischaemic damage in rodent models, with the absence of IL- $1 \alpha$ or IL- $1 \beta$ in knockout mice ameliorating damage [43]. A recent meta-analysis showed that IL$1 \mathrm{Ra}$ administration was associated with a $38.2 \%$ reduction in mean infarct volume across 16 published preclinical studies [44]. It has been hypothesized that the inhibition of IL- $1 \beta$ generated centrally following an acute ischemic stroke would reduce further cerebral injury mediated by inflammation. IL-1 receptor antagonist has long been available for clinical use in arthritis [45]. However, clinical development of IL-1 receptor antagonist after initial promising results in various cerebrovascular proof-of-concept trials, including ischemic stroke and subarachnoid haemorrhage, has been slowed by the replacement of the intravenous by a subcutaneously injected formula.

In a phase II study, 34 patients were block randomized to receive either recombinant human IL-1 receptor antagonist (rhIL-1Ra; Anakinra) administered intravenously with a 100-mg loading dose over $60 \mathrm{~s}$, followed by a $2 \mathrm{mg} / \mathrm{kg} / \mathrm{h}$ infusion over $72 \mathrm{~h}$, or matching placebo, within $6 \mathrm{~h}$ of the onset of symptoms of acute stroke. No adverse events were attributed to treatment and the recombinant human IL-1Ra was deemed safe. Ready transfer across the blood-brain barrier was shown [46]. Furthermore, systemic markers of biological activity (including neutrophil and total white cell counts, Creactive protein, and IL-6 concentrations) were lower in the treatment arm. Clinical outcomes after at least 3 months were better in the treatment group. Median NIHSS score was reduced to 4 versus 1 in the placebo arm, and more patients receiving anakinra had $\mathrm{mRS}$ $0-1$ at 3 months (30\% vs $7 \%$ ) [46].

After the intravenous formulation of anakinra was discontinued an ongoing phase II randomized, controlled trial was started which investigated the effects of subcutaneous administration at doses of $100 \mathrm{mg}$ twice daily, administered for 3 days [47]. The primary outcome measure is reduction of inflammatory biomarkers (including IL-6) between $6 \mathrm{~h}$ and 5-7 days after stroke; secondary outcomes also include 3-month clinical outcomes (mRS, survival, and length of stay) $[6,47]$.

\section{Blockade of Neutrophils}

\section{Recombinant Neutrophil Inhibitory Factor}

Recombinant neutrophil inhibitory factor (UK-279, 276) is a recombinant glycoprotein with selective binding to the $\mathrm{CD} 11 \mathrm{~b}$ integrin of macrophage-1 antigen (CD11b/CD18) [48]. Demonstration of its ability to reduce neutrophil infiltration and infarct volume in rat models of stroke led to its consideration in humans [49].

The phase II clinical trial, "Acute Stroke Therapy by Inhibition of Neutrophils" (ASTIN), took a Bayesian sequential design allowing for double-blind, randomized, adaptive allocation to 1 of 16 dose tiers (range $10-120 \mathrm{mg}$ ) or placebo and early termination for efficacy or futility $[50,51]$. The primary endpoint was change from baseline to day 90 on the Scandinavian Stroke Scale (DeltaSSS), adjusted for baseline Scandinavian Stroke Scale. The study aimed for a 3-point additional mean recovery above placebo [50]. A total of 966 patients with stroke were included (887 ischemic infarcts, 204 co-treated with intravenous tissue plasminogen activator) and treated within $6 \mathrm{~h}$ of symptom onset. Though UK-279,276 was generally well tolerated, there was no dose-rate effect compared with placebo, and the trial was stopped early after inclusion of 966 patients with acute stroke [50].

\section{Neutrophil $\beta 2$ Integrin CD18}

Similar to the mechanism underpinning Recombinant neutrophil inhibitory factor, use of the humanized monoclonal antibody against neutrophil $\beta 2$ integrin CD18 (Hu23F2G) was hypothesized to improve long-term outcomes after ischemic stroke [6]. The safety and dose efficacy of this approach was investigated in a phase II dose-escalation study, where Hu23F2G was administered within $12 \mathrm{~h}$ of ictus $[6,49]$. A concentration of $1.5 \mathrm{mg} / \mathrm{kg} \mathrm{Hu} 23 \mathrm{~F} 2 \mathrm{G}$ in single dose was found to be generally safe, though noted to increase fever. Furthermore, twice-daily infusion of this dose appeared to improve mRS. Consequently, a phase III study was performed. However, the study was terminated after the first interim analysis, when no likely benefit of treatment was observed [49]. Unfortunately, there has been no public information release about the results of the study, specifically the outcomes and safety issues [49].

\section{E-selectin}

E-selectin is a cell adhesion molecules expressed on endothelial cells that have been activated by inflammatory cytokines [52]. In humans, L-selectin is the main ligand, and with glycolipids constitutes more than half of the E-selectin receptors on neutrophils [53]. Although serum levels of E-selectin are not elevated following symptom onset in stroke patients [52], 
their systemic expression after focal cerebral ischaemia in animal models, along with the attenuation of ischaemic damage with transnasal administration has prompted clinical trials to explore their use in secondary prevention $[54,55]$. The hypothesis driving this work is that induction of mucosal tolerance may divert any inflammatory response from contributing to further cerebral insult. While the first of 2 studies investigating recombinant human E-selectin delivered intranasally was terminated prematurely [54] the second is currently ongoing in exploring the maximum safe dose in patients older than 45 years of age who have suffered an ischemic stroke or transient ischemic attack within 30-120 days [55]. Participants are randomly assigned to receive E-selectin at a dose level of 5,15 , or 50 micrograms or a placebo on alternate days for 5 doses, with this repeated a total of 3 times; followup is at 1 and 3 months [55].

\section{Intercellular Adhesion Molecule 1}

Enlimomab is a murine monoclonal antibody against intercellular adhesion molecule 1. Studies in rat models of acute ischemic stroke suggested improved neurological outcomes [56]. A consequent open, uncontrolled, dose titration study was undertaken in 32 patients hospitalized for ischemic or hemorrhagic stroke, with the aim of clarifying the safety, pharmacokinetics, and biological activity of enlimomab in this context [57]. Patients received 1 of 4 regimens of enlimomab; a loading dose infused within $24 \mathrm{~h}$ of symptom onset was followed by 4 daily maintenance doses, with the total dose ranging from 140 to $480 \mathrm{mg}$. The results suggested that doses of 140 to $480 \mathrm{mg}$ administered over 5 days did not increase the risk of adverse events during the observation period of 30 $( \pm 10)$ days [57]. Furthermore, the loading dose of $160 \mathrm{mg}$ followed by 4 daily maintenance doses of $40 \mathrm{mg}$ was deemed to be suitable for further study [57].

In a subsequent phase III study, 625 patients with ischemic stroke were randomized to receive 5 days of either enlimomab (317 patients) or placebo (308 patients) within $6 \mathrm{~h}$ of stroke symptom onset [58]. At day 90, mRS was worse in patients treated with enlimomab than with placebo. Fewer patients had symptom-free recovery on enlimomab, and more patients died [58]. These results were apparent on days 5, 30, and 90 of follow-up. Significantly more adverse events occurred in the enlimomab arm, most notably infections and fever [58]. Work in rodent models subsequently demonstrated that sequential infusion of heterologous antibodies following focal ischemia increaseS cerebral injury volume [59], suggesting that the detrimental effects of enlimomab may be attributable to the development of human antimouse antibodies, with consequent activation of neutrophils through complement-dependent mechanisms.

\section{Stroke Trials Targeting Adaptive Immune Cells}

The current concept is that lymphocytes exert their deleterious effects after entering the brain. Blockade of $\alpha 4-\beta 1$ integrin (also referred to as very late antigen 4 ) is effective in relapsing-remitting multiple sclerosis (MS), and is believed to mediate its effect by restricting leukocyte access through the blood-brain barrier, thus limiting neuroinflammation [60]. Such strategies may also be applicable to stroke. An alternative approach is to arrest lymphocyte egress from lymphatic organs. Again, this principle has been successfully applied in MS using the sphingosine receptor blocker fingolimod [61].

\section{Blocking of $\alpha 4-\beta 1$ Integrin}

Blockade of the $\alpha 4-\beta 1$ integrin on leukocytes is a potent strategy to attenuate neuroinflammation and to prevent relapses in MS. The recently completed ACTION study was a randomized controlled phase IIa trial comparing a single injection of $300 \mathrm{mg}$ of intravenous natalizumab against placebo within a 9-h time window after symptom onset $[62,63]$. Patients underwent serial magnetic resonance imaging (MRI) at baseline and on days 1, 5, and 30. Based on findings in rodent stroke models the trial intended to prove the concept that blockade of $\alpha 4$ integrin on leukocytes would prevent delayed infarct growth. Moreover, functional outcome parameters were determined While the trial found no effect of natalizumab on infarct growth between day 1 and day 5 (the primary study endpoint), patients receiving natalizumab were more likely to have an excellent clinical outcome at 30 and 90 days. This was particularly evident in subgroups of patients with smaller infarcts and with more exposure to the drug in pharmacokinetic area under the curve studies. No safety issues, including infectious complications, were noted.

The phase IIb ACTION trial has started in mid-2016 to test beneficial effects of 2 doses of natalizumab (single dose of $300 \mathrm{mg}$ and $600 \mathrm{mg}$, respectively) on functional outcome after stroke [64].

\section{Fingolimod}

The sphingosine 1-phosphate (S1P) lipid is secreted extracellularly following the metabolism of sphingomyelin from cell membrane structures. Signaling via G protein-coupled S1P receptors, it regulates a multitude of responses, including cell migration, differentiation, and survival [65]. Fingolimod (FTY720, Gilenya; Novartis, Basel, Switzerland) is an oral S1P receptor modulator that sequesters lymphocytes to lymph nodes and has been approved for therapy of relapsingremitting MS. It has more recently also been shown to be protective in a number of preclinical stroke studies [66]. 
In an open-label, 3-center pilot study enrolling 22 patients in China, fingolimod ( $0.5 \mathrm{mg} /$ day for 3 days $)$ on top of routine medical management or routine management alone was administered to patients within a time window of between at least $4.5 \mathrm{~h}$ and $72 \mathrm{~h}$ after symptom onset [67]. Candidates for intravenous thrombolysis were excluded. Fingolimod restricted enlargement of the infarct volume on sequential MRI between day 1 and day 7 and reduced blood-brain barrier permeability on contrast MRI [67]. The treatment was also associated with short-term neurological improvements, and no significant excess of adverse events was noted [67].

In a subsequent open pilot study by Zhu et al. [68], 47 patients were randomized in 3 centers to receive either fingolimod ( $0.5 \mathrm{mg}$ /day for 3 days) or nothing on top of intravenous thrombolysis. Again, patients receiving fingolimod had substantially less lesion volume growth at day 1 and day 7, less hemorrhagic transformation, and profoundly better outcomes on the mRS at 90 days. The effect of fingolimod was also tested in a pilot study of patients with intracerebral hemorrhage. In a 2-arm proof-of-concept clinical study, 11 participants were treated with $0.5 \mathrm{mg}$ oral fingolimod daily for 3 days after intracerebral hemorrhage; the first dose was administered within $72 \mathrm{~h}$ of the ictus [69]. Again, short- and long-term neurological functions were better in participants who received fingolimod than in participants who did not.

\section{Comparison of Natalizumab and Fingolimod}

The discrepancy between the impact of natalizumab and fingolimod on imaging endpoints in early phase II stroke trials is striking. Although both drugs have T lymphocytes as the presumed key target immune cell population, their effects on early infarct growth differed markedly. First, the sample size of trials was small, and findings should be considered preliminary for both drugs. Second, the trials with fingolimod were open investigator-initiated studies, which implies a different regulatory environment. Third, the ACTION trial was performed predominantly in caucasian patients, whereas the fingolimod trials enrolled only South-East Asian patients. Leaving these formal differences among trials aside, the discrepancy of outcomes may point to different biological effects of both drugs. For example, S1P receptors are also present on other brain cells, and there is some evidence suggesting that direct effects of fingolimod on astrocytes and neurons may be protective. Fingolimod reduces lymphocytes in the blood, which may have beneficial effects even before brain invasion. Finally, fingolimod was repetitively given over 3 days with a cease of action within days, whereas natalizumab blocks the $\alpha-4$ integrin for at least 4 weeks.

Although both deleterious and protective regulatory roles of B lymphocytes are increasingly recognized, translation into clinical trials in stroke has not happened yet.

\section{Future Directions}

There is little doubt now that immune mechanisms profoundly affect the pathophysiology in the ischemic brain. Previous trials of immunomodulatory therapies have focused on attenuating the detrimental effects of certain immune cells and inflammatory mediators on the extent of tissue damage in the acute phase. Despite solid evidence, including metaanalyses [44], and preclinical randomized trials for the effectiveness of this neuroprotective approach in experimental studies, the translational validity of neuroprotection by immune modulation is presently uncertain [70]. Findings in proof-of-concept neuroimaging studies in T lymphocytes are controversial and require further investigation. If confirmed, discrepancies between absent effects on the size of the macroscopic lesion and effects on functional outcomes in the ACTION trial may prompt a different conceptual approach towards the role of neuroinflammation in stroke in which inflammation causes more prolonged interference with brain function. A major limitation at present is a very limited understanding of the involvement of neuroinflammation in neurorepair and neurodegeneration.

A better understanding of the immune biology in experimental and human stroke is needed to identify additional targets for early and delayed interventions. However, present data suggest that the risks of immune modulation in the acute phase of stroke, including increased susceptibility to infections are limited. The growing number of immunomodulatory interventions that are already established for other indications in humans provides a unique opportunity to fast-track innovative proof-of-concept trials. Ideally, these trials are accompanied by surrogate marker studies, including molecular neuroimaging, to evaluate the translational validity of concepts derived from studies in animals. Extensive crosstalk between preclinical and clinical work is needed to elucidate the pathophysiology of the multifaceted poststroke immune response.

\section{Summary}

The current understanding of the immunological processes involved in brain injury and repair is still limited. Previous trials of immune modulation in stroke using IL-1 Ra and lymphocyte-targeted approaches have yielded some promising results, but confirmation in larger trials is needed. Conceptually, improving brain function by mitigating prolonged neuroinflammation triggered by acute brain injury could be an additional strategy to neuroprotection.

Required Author Forms Disclosure forms provided by the authors are available with the online version of this article. 


\section{References}

1. Emberson J, Lees KR, Lyden P, et al. Effect of treatment delay, age, and stroke severity on the effects of intravenous thrombolysis with alteplase for acute ischaemic stroke: a meta-analysis of individual patient data from randomised trials. Lancet 2014;384:1929-1935.

2. Campbell BC, Donnan GA, Lees KR, et al. Endovascular stent thrombectomy: the new standard of care for large vessel ischaemic stroke. Lancet Neurol 2015;14:846-854.

3. Chamorro A, Meisel A, Planas AM, Urra X, van de Beek D, Veltkamp R. The immunology of acute stroke. Nat Rev Neurol 2012;8:401-410.

4. Iadecola C, Anrather J. The immunology of stroke: from mechanisms to translation. Nat Med 2011;17:796-808.

5. Dirnagl U, Becker K, Meisel A. Preconditioning and tolerance against cerebral ischaemia: from experimental strategies to clinical use. Lancet Neurol 2009;8:398-412.

6. Smith CJ, Denes A, Tyrrell PJ, Di Napoli M. Phase II antiinflammatory and immune-modulating drugs for acute ischaemic stroke. Exp Opin Invest Drugs 2015;24:623-643.

7. Fu Y, Liu Q, Anrather J, Shi FD. Immune interventions in stroke. Nat Rev Neurol 2015;11:524-535.

8. Gill D, Veltkamp R. Dynamics of T cell responses after stroke. Curr Opin Pharmacol 2016;26:26-32.

9. Chamorro A, Urra X, Planas AM. Infection after acute ischemic stroke: a manifestation of brain-induced immunodepression. Stroke 2007;38:1097-1103

10. Klehmet J, Harms H, Richter M, et al. Stroke-induced immunodepression and post-stroke infections: lessons from the preventive antibacterial therapy in stroke trial. Neuroscience 2009;158:1184-1193

11. Meisel C, Schwab JM, Prass K, Meisel A, Dirnagl U. Central nervous system injury-induced immune deficiency syndrome. Nat Rev Neurosci 2005;6:775-786.

12. Prass K, Meisel C, Hoflich C, Braun J, Halle E, Wolf T, et al. Stroke-induced immunodeficiency promotes spontaneous bacterial infections and is mediated by sympathetic activation reversal by poststroke T helper cell type 1-like immunostimulation. J Exp Med 2003;198:725-736.

13. Mracsko E, Veltkamp R. Neuroinflammation after intracerebral hemorrhage. Front Cell Neurosci 2014;8:388.

14. Shichita T, Ito M, Yoshimura A. Post-ischemic inflammation regulates neural damage and protection. Front Cell Neurosci 2014;8: 319.

15. da Fonseca AC, Matias D, Garcia C, et al. The impact of microglial activation on blood-brain barrier in brain diseases. Front Cell Neurosci 2014;8:362.

16. Kuric E, Ruscher K. Dynamics of major histocompatibility complex class II-positive cells in the postischemic brain-influence of levodopa treatment. J Neuroinflammation 2014;11:145.

17. Schoknecht K, David Y, Heinemann U. The blood-brain barriergatekeeper to neuronal homeostasis: clinical implications in the setting of stroke. Semin Cell Develop Biol 2015;38:35-42.

18. Engelhardt S, Huang SF, Patkar S, Gassmann M, Ogunshola OO. Differential responses of blood-brain barrier associated cells to hypoxia and ischemia: a comparative study. Fluids Barriers CNS 2015;12:4

19. Mracsko E, Javidi E, Na SY, Kahn A, Liesz A, Veltkamp R. Leukocyte invasion of the brain after experimental intracerebral hemorrhage in mice. Stroke 2014;45:2107-2114

20. Chu HX, Kim HA, Lee S, et al. Immune cell infiltration in malignant middle cerebral artery infarction: comparison with transient cerebral ischemia. J Cereb Blood Flow Metab 2014;34:450-459.
21. Gelderblom M, Leypoldt F, Steinbach K, et al. Temporal and spatial dynamics of cerebral immune cell accumulation in stroke. Stroke 2009;40:1849-1857.

22. Jin R, Yang G, Li G. Inflammatory mechanisms in ischemic stroke: role of inflammatory cells. J Leukoc Biol 2010;87:779-789.

23. Yilmaz G, Arumugam TV, Stokes KY, Granger DN. Role of T lymphocytes and interferon-gamma in ischemic stroke. Circulation 2006;113:2105-2112.

24. Liesz A, Suri-Payer E, Veltkamp C, et al. Regulatory T cells are key cerebroprotective immunomodulators in acute experimental stroke. Nat Med 2009;15:192-199.

25. Liesz A, Zhou W, Mracsko E, et al. Inhibition of lymphocyte trafficking shields the brain against deleterious neuroinflammation after stroke. Brain 2011;134:704-720.

26. Kleinschnitz C, Schwab N, Kraft P, et al. Early detrimental T-cell effects in experimental cerebral ischemia are neither related to adaptive immunity nor thrombus formation. Blood 2010;115:38353842.

27. Mracsko E, Liesz A, Stojanovic A, et al. Antigen dependently activated cluster of differentiation 8-positive $\mathrm{T}$ cells cause perforinmediated neurotoxicity in experimental stroke. J Neurosci 2014;34: 16784-16795.

28. Shichita T, Sugiyama Y, Ooboshi H, et al. Pivotal role of cerebral interleukin-17-producing gammadeltaT cells in the delayed phase of ischemic brain injury. Nat Med 2009;15:946-950.

29. Gelderblom M, Weymar A, Bernreuther C, et al. Neutralization of the IL-17 axis diminishes neutrophil invasion and protects from ischemic stroke. Blood 2012;120:3793-3802.

30. Ren X, Akiyoshi K, Dziennis S, et al. Regulatory B cells limit CNS inflammation and neurologic deficits in murine experimental stroke. J Neurosci 2011;31:8556-8563.

31. Doll DN, Barr TL, Simpkins JW. Cytokines: their role in stroke and potential use as biomarkers and therapeutic targets. Aging Dis 2014;5:294-306.

32. Seifert HA, Collier LA, Chapman CB, Benkovic SA, Willing AE, Pennypacker KR. Pro-inflammatory interferon gamma signaling is directly associated with stroke induced neurodegeneration. J Neuroimmune Pharmacol 2014;9:679-689.

33. Gelderblom M, Arunachalam P, Magnus T. gammadelta T cells as early sensors of tissue damage and mediators of secondary neurodegeneration. Front Cell Neurosci 2014;8:368.

34. Peruzzotti-Jametti L, Donega M, Giusto E, Mallucci G, Marchetti $\mathrm{B}$, Pluchino S. The role of the immune system in central nervous system plasticity after acute injury. Neuroscience 2014;283:210221.

35. Doyle KP, Quach LN, Sole M, et al. B-lymphocyte-mediated delayed cognitive impairment following stroke. J Neurosci 2015;35: 2133-2145.

36. Lampl Y, Boaz M, Gilad R, et al. Minocycline treatment in acute stroke: an open-label, evaluator-blinded study. Neurology 2007;69: 1404-1410.

37. Fagan SC, Waller JL, Nichols FT, et al. Minocycline to improve neurologic outcome in stroke (MINOS): a dose-finding study. Stroke 2010;41:2283-2287.

38. Singhealth Foundation. Neuroprotection With Minocycline Therapy for Acute Stroke Recovery Trial (NeuMAST). In: ClinicalTrials.gov [Internet]. Bethesda (MD): National Library of Medicine (US). 2000- [cited 2016 May 21]. Available from: http://clinicaltrials.gov/show/NCT00930020

39. Andrews AE, Barcham GJ, Brandon MR, Nash AD. Molecular cloning and characterization of ovine IL-1 alpha and IL-1 beta. Immunology 1991;74:453-460.

40. Dinarello CA. A clinical perspective of IL-1beta as the gatekeeper of inflammation. Eur J Immunol 2011;41:1203-1217. 
41. Wang X, Barone FC, Aiyar NV, Feuerstein GZ. Interleukin-1 receptor and receptor antagonist gene expression after focal stroke in rats. Stroke 1997;28:155-161.

42. Luheshi NM, Kovacs KJ, Lopez-Castejon G, Brough D, Denes A. Interleukin-1alpha expression precedes IL-1beta after ischemic brain injury and is localised to areas of focal neuronal loss and penumbral tissues. J Neuroinflammation 2011;8:186.

43. Murray KN, Parry-Jones AR, Allan SM. Interleukin-1 and acute brain injury. Front Cell Neurosci 2015;9:18.

44. Banwell V, Sena ES, Macleod MR. Systematic review and stratified meta-analysis of the efficacy of interleukin-1 receptor antagonist in animal models of stroke. J Stroke Cerebrovasc Dis 2009;18:269276.

45. Rothwell N, Allan S, Toulmond S. The role of interleukin 1 in acute neurodegeneration and stroke: pathophysiological and therapeutic implications. J Clin Invest 1997;100:2648-2652.

46. Emsley HC, Smith CJ, Georgiou RF, et al. A randomised phase II study of interleukin-1 receptor antagonist in acute stroke patients. J Neurol Neurosurg Psychiatry 2005;76:1366-1372.

47. Brain Injury Research Team; Hope Hospital (UK). Subcutaneous interleukin-1 receptor antagonist (SC IL-1RA) in Stroke Study. In: isrctn.com [Internet]. BioMed Central. 2000- [cited 2016 May 21]. Available from: http://www.isrctn.com/ISRCTN74236229 ISRCTN Identifier: ISRCTN74236229.

48. Muchowski PJ, Zhang L, Chang ER, Soule HR, Plow EF, Moyle $M$. Functional interaction between the integrin antagonist neutrophil inhibitory factor and the I domain of CD11b/CD18. J Biol Chem 1994;269:26419-26423.

49. del Zoppo GJ. Acute anti-inflammatory approaches to ischemic stroke. Ann N Y Acad Sci 2010;1207:143-148.

50. Krams M, Lees KR, Hacke W, et al. Acute Stroke Therapy by Inhibition of Neutrophils (ASTIN): an adaptive dose-response study of UK-279,276 in acute ischemic stroke. Stroke 2003;34: 2543-2548.

51. Grieve AP, Krams M. ASTIN: a Bayesian adaptive dose-response trial in acute stroke. Clin Trials 2005;2:340-351.

52. Shyu KG, Chang H, Lin CC. Serum levels of intercellular adhesion molecule-1 and E-selectin in patients with acute ischaemic stroke. J Neurol 1997;244:90-93.

53. Chase SD, Magnani JL, Simon SI. E-selectin ligands as mechanosensitive receptors on neutrophils in health and disease. Ann Biomed Eng 2012;40:849-859.

54. National Institute of Neurological Disorders and Stroke (NINDS). E-Selectin Nasal Spray to Prevent Stroke Recurrence. In: ClinicalTrials.gov [Internet]. Bethesda (MD): National Library of Medicine (US). 2000- [cited 2016 May 21]. Available from: $\mathrm{http}: / /$ clinicaltrials.gov/show/NCT00012454

55. National Institute of Neurological Disorders and Stroke (NINDS). E-Selectin Nasal Instillation to Prevent Secondary Stroke. In: ClinicalTrials.gov [Internet]. Bethesda (MD): National Library of Medicine (US). 2000- [cited 2016 May 21]. Available from: http:/clinicaltrials.gov/show/NCT00069069
56. Zhang RL, Chopp M, Li Y, et al. Anti-ICAM-1 antibody reduces ischemic cell damage after transient middle cerebral artery occlusion in the rat. Neurology 1994;44:1747-1751.

57. Schneider D, Berrouschot J, Brandt T, et al. Safety, pharmacokinetics and biological activity of enlimomab (anti-ICAM-1 antibody): an open-label, dose escalation study in patients hospitalized for acute stroke. Eur Neurol 1998;40:78-83.

58. Enlimomab Acute Stroke Trial I. Use of anti-ICAM-1 therapy in ischemic stroke: results of the Enlimomab Acute Stroke Trial. Neurology 2001;57:1428-1434.

59. Furuya K, Takeda H, Azhar S, et al. Examination of several potential mechanisms for the negative outcome in a clinical stroke trial of enlimomab, a murine anti-human intercellular adhesion molecule-1 antibody: a bedside-to-bench study. Stroke 2001;32:2665-2674.

60. Engelhardt B, Kappos L. Natalizumab: targeting alpha4-integrins in multiple sclerosis. Neurodegener Dis 2008;5:16-22.

61. La Mantia L, Tramacere I, Firwana B, Pacchetti I, Palumbo R, Filippini G. Fingolimod for relapsing-remitting multiple sclerosis. Cochrane Database Syst Rev 2016;4:CD009371.

62. Elkins J, Elkind M, Veltkamp R, et al. Natalizumab Versus Placebo in Patients with Acute Ischemic Stroke (AIS): Results from ACTION, a Multicenter, Double-Blind, Placebo-Controlled, Randomized Phase 2 Clinical Trial (S7.005). Neurology 2016;86(16 Supplement).

63. Biogen. Effect of Natalizumab on Infarct Volume in Acute Ischemic Stroke (ACTION). In: ClinicalTrials.gov [Internet]. Bethesda (MD): National Library of Medicine (US). 2000- [cited 2016 May 21]. Available from: https://clinicaltrials.gov/ct2/show/NCT01955707

64. Biogen. Safety and Efficacy of Intravenous Natalizumab in Acute Ischemic Stroke (ACTION II). In: ClinicalTrials.gov [Internet]. Bethesda (MD): National Library of Medicine (US). 2000- [cited 2016 May 21]. Available from: http://clinicaltrials. gov/show/NCT02730455

65. Mendelson K, Evans T, Hla T. Sphingosine 1-phosphate signalling. Development 2014;141:5-9.

66. Brunkhorst R, Vutukuri R, Pfeilschifter W. Fingolimod for the treatment of neurological diseases-state of play and future perspectives. Front Cell Neurosci 2014;8:283.

67. Fu Y, Zhang N, Ren L, et al. Impact of an immune modulator fingolimod on acute ischemic stroke. Proc Natl Acad Sci U S A 2014;111:18315-18320

68. Zhu Z, Fu Y, Tian D, et al. Combination of the immune modulator fingolimod with alteplase in acute ischemic stroke: a pilot trial. Circulation 2015;132:1104-1112.

69. Fu Y, Hao J, Zhang N, et al. Fingolimod for the treatment of intracerebral hemorrhage: a 2-arm proof-of-concept study. JAMA Neurol 2014;71:1092-1101.

70. Llovera G, Hofmann K, Roth S, et al. Results of a preclinical randomized controlled multicenter trial (pRCT): Anti-CD49d treatment for acute brain ischemia. Sci Transl Med 2015;7:299ra121. 